\title{
On the New Boson Higgs's Studies at the CERN-ATLAS Experiment. The Emergency of a Historical Discovery
}

\author{
Raffaele Pisano ${ }^{1,2}$ \\ ${ }^{1}$ Sciences, Sociétés, Cultures dans leurs Evolutions, University of Lille 1, Lille, France \\ ${ }^{2}$ Research Center for Theory and History of Science, University of West Bohemia, Plzen, Czech Republic \\ Email: pisanoraffaele@iol.it
}

Received December $23^{\text {rd }}, 2012$; revised January $25^{\text {th }}, 2013$; accepted February $5^{\text {th }}, 2013$

\begin{abstract}
This paper is a summary of the interview-workshop to Aleandro Nisati (12 December 2012, SEMMService Enseignement et Multimédia) co-organized by UFR Physique, University of Lille 1, France (Raffaele Pisano, Remi Franckowiak, Bernard Maitte and Lisa Rougetet), ATLAS Experiment Team (CERN, Genève, Switzerland), in persons of the cited Italian scientist-already Physics coordinator at ATLAS - and his colleague, Steven Goldfarb (CERN-University of Michigan, USA). The latter kindly answered to the questions on the ATLAS detector, LHC machine and CERN-ATLAS laboratories proposed by the participants. Distinguished lectures by historians of science at University of Lille 1 (Bernard Maitte, Bernard Pourprix and Robert Locqueneux) specialist on history of physics opened the workshop session.
\end{abstract}

Keywords: History of Physics; Science and Society; Standard Model Higgs Boson

\section{A Short Introduction on the Discovery}

On July, 4th 2012, the ATLAS experiment presented a preview of its updated results on the search for the Higgs Boson. (Figure 1). The results were shown at a seminar held jointly at CERN and via video link at ICHEP, the International Conference for High Energy Physics in Melbourne, Australia (Atlas Collaboration, 2012; CMS Collaboration, 2012). At CERN, preliminary results were presented to scientists on site and via webcast to colleagues located in hundreds of institutions around the world. Aleandro Nisati was ATLAS Physics coordinator.

The Higgs boson is the only missing elementary particle of the Standard Model (SM) of particles and fields. In the SM, the non-zero vacuum expectation value of the Higgs field breaks the electroweak gauge symmetry. It is the simplest process capable of giving mass to the gauge bosons and elementary fermions. Its quantum would be a scalar boson, the only one in this theory. A brief overview of the searches for this particle with the A Toroidal LHC ApparatuS (ATLAS) detector at the Large Hadron Collider (LHC) is given. In particular, the latest results of the search for this particle at the LHC are summarized and discussed, focusing on the recent observation of a new boson by the experiments ATLAS and Compact Muon Solenoid (CMS, the main experiments) at the LHC, with a mass around $126 \mathrm{GeV}$. Preliminary available data show that this particle is consistent with the boson predicted by the SM. More data are needed to perform precision measurements of the physics properties of this new boson, and verify whether this is the Higgs boson predicted by Standard Model.

\section{Interview to Aleandro Nisati}

The latest results concerning the discovery of a new boson (m $\sim 126 \mathrm{GeV}$ ) were experimented by A Toroidal LHC Appa-

*Short review.
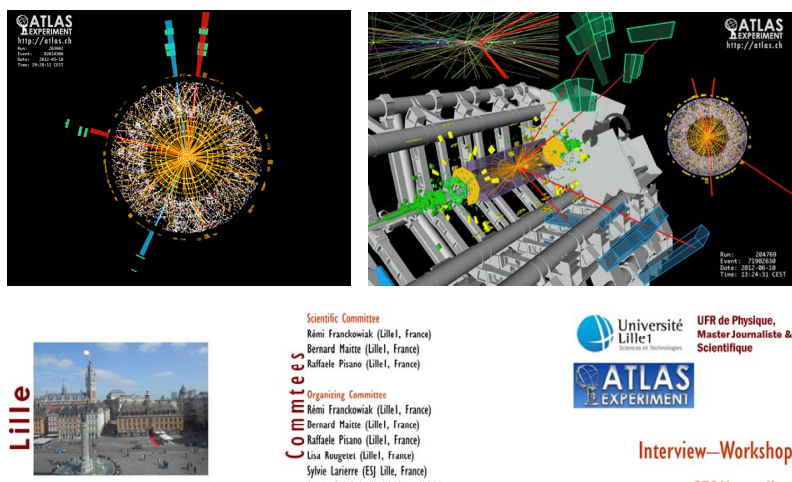

Université UfrR de Physique,
Mastorsournalicto
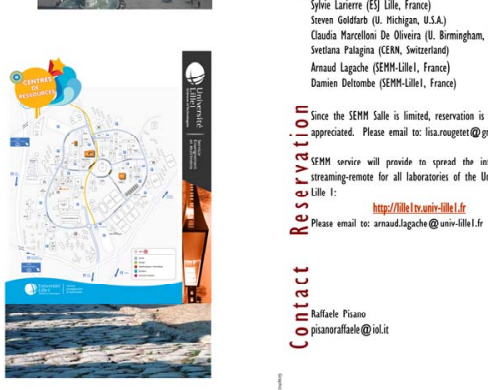

CERN on line

Aleandro Nisati:

On Higgs' Boson

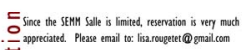

AILAS Experiment

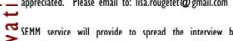

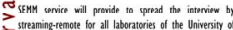
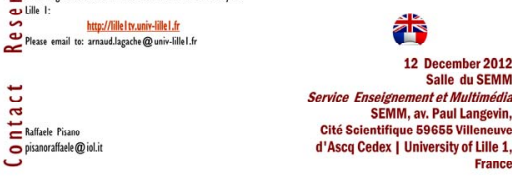

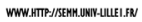
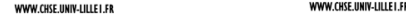

Figure 1.

Higgs decay to four electrons recorded by ATLAS in 2012 (Bottom). Higgs decay to four muons recorded by ATLAS in 2012. Images credit: URL (last checked 14 January 2013). http://www.atlas.ch/news/2012/ latest-results-from-higgs-search.html. Nisati Workshop Brochure organized at University of Lille 1, France.

ratuS (ATLAS) and Compact Muon Solenoid (CMS) at the Large Hadron Collider (LHC) and recently exposed at SISFA 2012 Congress where I interviewed Aleandro Nisati (I.N.F.N. Sezione di Roma-CERN, Italy/Switzerland), ATLAS Physics co- 
ordinator who had a major role in the discovery and key-note at the Congress. This new particle is effetely consistent, within the current available experimental accuracy, with the Standard Model Higgs boson (SMHB).

But, what is the importance of the Higgs boson?

Nisati: the Higgs boson is the only missing elementary particle of the Standard Model (SM) of particles and fields. Here the non-zero vacuum expectation value of the Higgs field breaks the electroweak gauge symmetry. It is the simplest process capable of giving mass to the gauge bosons and fermions.

What about preliminary available experimental data?

Nisati: the quantum associated to this field is a spin- 0 particle, the Higgs boson. An indirect constraint on the Higgs boson mass of $\mathrm{m}_{\mathrm{H}}<185 \mathrm{GeV}$ at $95 \%$ confidence level (CL) has been set using global fits to electroweak precision data. Direct searches (up to 2011) excluded at 95\% CL a SMHB with mass $\mathrm{m}_{\mathrm{H}}<114.4 \mathrm{GeV}$ and $147<\mathrm{m}_{\mathrm{H}}<179$ respectively. The search for this particle was pursued at the LCH looking in particular to high mass resolution channels: the diphoton and the 4-lepton final states. The data sample used in the analysis was based on about $5 \mathrm{fb}^{-1}$ of proton-proton collisions data taken at $\sqrt{\mathrm{s}}=7$ $\mathrm{TeV}(2011)$ and $5.5 \mathrm{fb}^{-1}$ taken at $\sqrt{\mathrm{s}}=8 \mathrm{TeV}$ data (early 2012). In the diphoton final state, both ATLAS and CMS observed an excess of events around the $\gamma \gamma$ invariant mass of 125 $126 \mathrm{GeV}$, on top of a smooth background produced mainly by SM $\gamma \gamma$ process. Jet-jet and $\gamma$-jet processes, a potentially dangerous background with jets misidentified as photons, are suppressed thanks the robust photon identification and reconstruction provided by the high-performance electromagnetic calorimeters of these two experiments. An excess of events is observed also in the $\mathrm{H} \rightarrow \mathrm{ZZ} * \rightarrow$ 4-lepton channel (where for leptons only electrons and muons are considered). In this case, the dominant background is represented by the SM diboson production $Z^{*} \rightarrow 4$-leptons (irreducible background) and by $\mathrm{Z}+$ jets processes, where jets can be mis-reconstructed as electrons or muons. Also in this case, the robust electron and muon identification in both experiments allows a strong reduction of $\mathrm{Z}+$ jets events below the irreducible background. Finally, this excess is observed also in the low mass resolution channel $\mathrm{H} \rightarrow \mathrm{WW}^{*} \rightarrow \mathrm{lvl} \mathrm{v}$, in a mass interval fully consistent with the $125-126 \mathrm{GeV}$ mass, where the excess is observed in $\gamma \gamma$ and 4-leptons. The statistical combination of these results for ATLAS, and independently for CMS, leads to the observation of an excess of events at around $125 \mathrm{GeV}$ mass with at least 5 -sigma significance (corresponding to a probability $\sim 4 \times 10^{-7}$ ) per experiment.

Are these results well-matched with historical theoretical theory hypothesed by Higgs last century?

Nisati: at this stage the results are compatible with the hypothesis that the new particle is the Higgs boson predicted by the SM. We have to wait to claim that the boson discovered is exactly Higgs Boson; particularly, observing this new particle also in final states with fermions, such as $\mathrm{H} \rightarrow \tau \tau$ and $\mathrm{H} \rightarrow$ b-bbar.

What is the main consequence if Higgs boson is confirmed?

Nisati: in case, SM will receive one of the strongest support from experimental results. On the contrary, deviations of this particle from the Standard Model Higgs boson will inevitably indicate new physics at the energy scale of the LHC. In both cases, a new extraordinary and exciting era in particle physics just opened up.

\section{Conclusion}

Thus, is seems that, more data are needed to perform precision measurements of the physics properties of this new boson, and verify whether this is the Higgs boson predicted by Standard Model.

Pisano: Maybe new reflections on the history of the World and its live components might be near to have a crucial founded hypothesis?

\section{Acknowledgements}

I would like to express my sincere gratitude to Aleandro Nisati for his friendly and precious collaboration. I also thank Bernard Maitte, Remi Franckowiak, Bernard Pourprix, Robert Locqueneux, and Lisa Rougetet for co-authoring in the workshop-interview and further process during workshop organizing terms.

\section{REFERENCES}

ATLAS Collaboration (2012). Observation of a new particle in the search for the standard model higgs boson with ATLAS detector at the LHC. Physics Letter B 716, 1, 1-29. URL (last checked 14 January 2013).

http://www.sciencedirect.com/science/article/pii/S037026931200857X

CMS Collaboration (2012). Observation of a new boson at a mass of $125 \mathrm{GeV}$ with the CMS experiment at the LHC. Physics Letter B, 716, 30-61. URL (last checked 14 January 2013).

http://www.sciencedirect.com/science/article/pii/S037026931200858

Higgs, P. W. (1964). Broken symmetries and the masses of Gauge Bosons. Physics Review Letter, 13, 508-509. doi:10.1103/PhysRevLett.13.508

Higgs, P. W. (1966). Spontaneous symmetry breakdown without Mass less Bosons. Physics Review Letter, 145, 1156-1163.

\section{READINGS}

Lévy-Leblond, J. M. (1996). Aux contraires, l'exercice de la pensée et la pratique de la science. NRF Essais, Paris: Gallimard.

Lévy-Leblond, J. M. (2006). De la matière: Relativiste, quantique, interactive. Paris: Seuil, Traces Écrites.

Locqueneux, R. (2009). Une histoire des idées en physique. SFHST, Paris: Vuibert.

Kibble, T. W. (1967). Symmetry breaking in non-abelian gauge theories. Physics Review Letter, 145, 1554-1561.

Pisano, R., \& Casolaro, F. (2012). A historical inquiry on geometry in relativity. Reflections on late relationship geometry-physics (Part Two). History Research, 2, 56-64.

Pourprix, B. (2009). D’où vient la physique quantique? Paris: Vuibert et Adapt.

Pourprix, B. (2010). La naissance de la physique quantique: Rupture et continuité. Bulletin de l'union des professeurs de physique et de chimie, 104, 1037-1050.

Pourprix, B. (2013). La genèse de l'atome de Bohr (forthcoming). Images de la physique 2012. Paris: CNRS.

The ALEPH, DELPHI, L3, OPAL, SLD, CDF and D0 Collaborations (2010). Precision electroweak measurements and constraints on the standard model. CERN-PH-EP-2010-095. 


\section{Appendix}

\section{Notes on Aleandro Nisati}

Aleandro Nisati is I.N.F.N. (The Italian Institute of Nuclear Physics) physicist researcher and scientific associate at CERN on LHC (Large Hadron Collider), Geneva. His research regards with new and strange particles producing a large publishing-and-spreading-job within the ECFA (The European Committee for Future Accelerators) particularly on Higgs searches, as well as studies of muon production, in proton-proton collisions at the LHC. He is one of the main founding physicists of one of the two main experiments at LHC, A Toroidal LHC ApparatuS (ATLAS) where he has been recently Physics Experi- mental Coordinator: scientific program and the project on muon detection and spectrometer, trigger system. Nisati also designed the first-level muon trigger algorithm, as well as the one of the second-level and for that he was elected chair of the Trigger/DAQ Institutes Board until 2007, and Higgs group coconvener for next two years. Recently (2012) he is also coordinator of the "ATLAS Input to the European Strategy Preparatory Group". ATLAS (and CMS, the main experiments at LHC) has found in summer 2012 a strong evidence of the production at the LHC of a new boson with mass near $126 \mathrm{GeV}$. This new particle is consistent, within the current available experimental accuracy, with the Standard Model Higgs boson. 